\title{
Rastreabilidade das Medições de pH - Parte II pH e a Junção Líquida
}

\author{
MARIA JOSE FERREIRA REBELO*
}

Recentemente, n 76 JanciroMarço 2000, foi feita uma aprescrntação deste assunto. pclo que tudo o que diz respeito à noçâo da plf c atribuiçăo de um valur de pif pelo método de Harnéd a urn padrăo prí mário pode ser revista nesse artigo. Foi entăo referido que a auribuição de pil a padrōes sccundários cra fejta por células comparalivas de Pt. $\mathrm{H}_{7}$, com junçào líquida. Apresenta-se agora uma cólula comparativa que permike obler valores de pll com a rastrabilidade exigida

\section{MEDIDA DO $\mathrm{pH}$ E POTENCIAI DE JUNÇÄO LÍQUIDA RESIDUAL}

Sempre que, num laboratório. no canno ou em qualquer outra situaçāo, sé pretende medir o valor de $\mathrm{pH} d e^{2}$ uma dada amostra ou estudar at sua variaçà recorre-sc a uma comparaçào com soluçōes de $\mathrm{pH}$ conhecido. Ou scja a medida de pH é uma médida comparałiva. Há que calibrar previamente o aparelho medidor de $\mathrm{pH}$ com. pelo incmos, uma solução de $\mathrm{pH}$ conhecido. Recorre-se entăo a uma medida do potencial da célula

Eléctrodo de referência I KCl (sat.) || Solução tampão (5) | eléctrodo de vidru

(Onde $S$ se refere a um padrăo primário ou secundário ) a que se stgue outra medida com a soluçäo anuostra em lugar do tampão. $\mathrm{OpH}$ é atribuido à solução amosira - X - pcla ex pressàa

$$
\Gamma \mathrm{H}(\mathrm{X})=\mathrm{pH}(\mathrm{S})+\frac{\mathrm{E}(\mathrm{S})-\mathrm{E}(\mathrm{X})}{\mathrm{RT} / \mathrm{F} \ln 0} \quad \mathrm{I}
$$

Ao fazer-se este cálculo do pli inclui-se no seu valor um potencial de junçăo líquida residual convencional. Com cléto o potencial da cé lula I inclui uma contribuiça para o porencial devida ao potencial de junçào líquida que sc estabelece entre o $\mathrm{KCl}$ é a soluçào de pH a medir, qua ć difurente para diferemtes soluçōes. Assim o verdadeiro $\mathrm{pH}$ como base de Mélodos Sccundários".

devia ser obtido por:

$$
\begin{aligned}
& \mathrm{pH}(X)=\mathrm{pH}(S)+\frac{E(S)-E(X)}{\mathrm{RT} / \mathrm{F} \operatorname{In} 0}+ \\
& +\frac{\mathrm{E}_{\mathrm{J}}(S)-E_{(X)}(X)}{\mathrm{RT} / \mathrm{E} \ln 0}
\end{aligned}
$$

onde $\mathrm{E}_{\jmath}(5)$ t́ o potencial de junçào líquida cntre o $\mathrm{KCl}$ e o padrăo e $\mathrm{E}_{\mathrm{T}}(\mathrm{X})$ entrc $\mathrm{KCl}$ e a solução amostra. Além disso o valor atribuido ao $\mathrm{pH}$ pclo método primário - que utiliza a célula de Harned - vem afectądo de ligeiras inconsistências, devidas à convenção de Bates Gugycinluim usada na equaça do coeficiente de actividade do iăo clorèo [1].

\section{ATRIBUIÇÄO DE UM V ALOR DE PH A UMA SOLUÇÃO PADRÃO}

Como já foi reférido, a atribuíçăo de um valor de pH a um tampào prinuário á feita pelo método de Iarned - quc cnvolve una célula sem junçăo líquida. Foi tambén indicado que "várias células que permitam medir a diference de $\mathrm{pH}$ entre duas soluçòcs tampào tềm sido propostas

Com efeílo, a célula cujo design se apresenta na fig. I foi desenvolvidröes secundärios pela autora.

Esta célula permite fazer, numa só operação o que, na práuica corrente sc faz cm duas operaçöes, isto é comparar o valor do pll de uma soluçăo desconhecida com 0 de um tampão padrāo. O êxito alcançado por este design pode ser avaliado pelo facto de a autora ter merecido o prémio Wynne Jones em I981. com o trabalho "Improvements in pli measurements" quc envolvia o desenvolvimento do design e a atribuiçăo de valotes de pli a uma série da para atribuir valores de $\mathrm{pH}$ a pa- de padrōes secundários com a mesma célula . tendo os valores obti dos sido posteriomente publicados. inicialmente na Tese de Doutoramento [2] e apenas como uma relerência em [3], seguidamente surgiram em tabelas em [4], finalmente a publicaça pormenorizada do trabalho aparcocu cm [5] e novamente reforidos com [6]. Nesta célula a. junçocs líquidas săo formadas cm tubos capilares de simetria cilindrica. de grande reproduibilidade. Por ter uste tipo de junçăo e tamb́rín porque apenas há necessidade de se recorrer a uma determinaçăo para atriłuir um valor de pll a urna solução este é um Método Sccundário por excelência. (De rotar ainda que o uso desta célula para atribuir valores dc $\mathrm{pH}$ a soluçōes tampăo de referéncia tcm as vantagens, relativamontc ao método de Harned, de prascindir de: $u s o$ do elécurodo de prata/cloreto de prata, adição de cloreto de sódio ao tampäo em estudo le consegucnes medidas em triplicado. no mínima\}, da extrapolaçăo para $\mathrm{mal}_{\mathrm{a}}=0$, do uso da convenção de Bates-Guggenlucirn para o $\gamma \mathrm{Cl}$, diminumdo assirn a incerteza associa da ao valor final)

Nesta célula, para cujo manuseamento se remete o lcitor para a ref [5]. introduzen-sc dois eléctrodos de Platina platinuada, ou paladizada, se se usar hidrogenoílalato do polássio. em cada um dos compartimentos lolerais A, A enquanto no central, B. se coloca KCl saturado que também enche a motade inferior dos tubos capilares dos compartimentos laterais. Faz-sc dcpois borbu lhar hidrogénio quc passa previamente sum pre-saturador antes de entrar na célula em C. C c c sai desta pelos tubos D. D' que se ligam a um outro exterior, cm Y. para lmat saida corlum. célula:

Mcde-se entäo o potencial da PL $\| H_{2}$ (g. I atmi | SS I KCl sat I PSI (g. I latm) $\mathrm{H}_{2}$ I Pt 


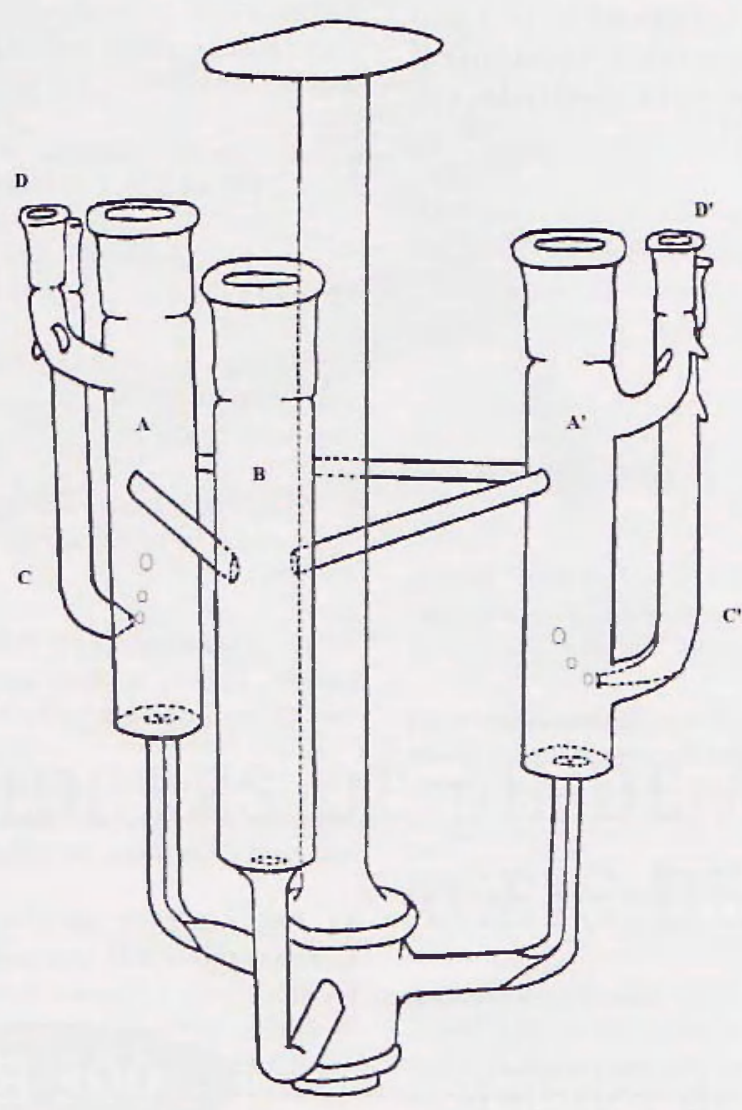

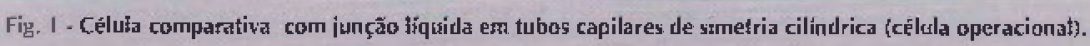

E o pH da solução tampão SS é dado por:

$$
\mathrm{pH}(\mathrm{SS})=\mathrm{pH}(\mathrm{PS})+\mathrm{E}_{\mathrm{II}} / \mathrm{RTl} 1110 \mathrm{~F}^{-1}
$$

onde PS representa, agora , o lampão primário e SS o secundário. Nesle valor do pll inclui-se, como já loî referido atrás, um potencial de junçà̃o líquida convencional, residual.

\section{CONVERSĂO DE ESCALAS DE $\mathrm{pH}$}

A utilizaçăo do hìdrogenoftalato de potássio como padrão primário e atribuição do $\mathrm{pH}$ a padrōes secundários pela célula Il com o design da autora (cl fig. 1) levou ao estabelecimento de uma escala de valores de $\mathrm{pH}$ atribuldos a padrōes secundários já pulblicada [3-6]. As diferenças entre os valores de pli atribuidos por esı método a alguns tampões considerados como padrö́cs sccundários te pelo método de Harned são devidas. como já se disse a um polenciai de junçào liquida residual convencional, variando os valores numa gama de milésimas a centésimas de $\mathrm{p}^{\mathrm{H}}$. Os valores mais elcvados da referida diferença observam-ste para os dois extremos da escala do $\mathrm{pH}$ c para Tris e fosfato de friperazina (cf fig.2 em [5|).

Se se usar un tampào primário diferente do hidrogenoftalato de potássio na célula II pode-se fazer uma interconversảo das escalas relativas aos diferentes padrōes primários. Isto é: seja o hidrogenoftalato de potássio o padrāo primário I - PSI e o lampảo fosfa to o padrão primário 2 - PS2 - Enıล๊o, o pIl atribuido a um padrăo secundário quando se uíliza o PS2 na célula II estará rclacionado con o que se oblém com o PS ] pela fórmula:

$$
\begin{aligned}
& \mathrm{pII}(S 5)_{\mathrm{TS2}}=\mathrm{pII}(\mathrm{SS})_{\mathrm{PSI}}+ \\
& \left.\div i \mathrm{pH}(\mathrm{PS} 2)-\mathrm{pH}(S S 2)_{\mathrm{PS} 1}\right)
\end{aligned}
$$

\section{JUNÇÃO LÍQUIDA DE SIMETRIA CILINDRICA E AS MEDIDAS DE pH EM: TAMPÖES PADRÃO. AGUAS DA CHUVA E DO MAR E SOLUÇÕES FISIOLÓGICAS}

As jumçōes formadas en tubos capilares com simetria cilindrica dāo gradientes de concentraçäo e potenciais cléctricos paralelos ao eixo do ubbo c, conscqucntemente, boa estabilidade e reprodutibilidade [7]. Assim aconteceu com esta célula ifig. 1) que foi também usada para outros sistemas $[8,91$. Um bom funcionamento da mesma requer uma boa qualidade da tomeira em Y usada para interligar os compartimenus [10].

Todas as medidas descritas at com forças iónicas próximas de 0.1. mol kg-1. Estudos fcitos com soluçóes a furças iónicas variando entre valores próximos do da água da chuva |I I] (I0-3 mol $\left.\mathrm{kg}^{-1}\right\}$ até os próximos da água do mar $(0,7$ mol $\left.\mathrm{kg}^{-1}\right)$ revelaram que us erros associados com capilares com $0.5 \mathrm{~mm}$ de diâmctro intemo eram inferiores a $0,2 \mathrm{mV}$ para soluçōes com força iónica superior a $\mathrm{I}^{-4} \mathrm{~mol} \mathrm{kg-1}^{-1}$ mas aumentavam para I mV para suluçōes $10^{-5} \mathrm{~mol} \mathrm{kg-1}$. Estes erros correspondem a diferenças de 0,0003 e 0.017 unjdades dc $\mathrm{pH}$ respectivamente. Também en sistemas fisiológicos, o método de referencia para as medidas de $\mathrm{pH}$ em sangue recomenda que se use $\mathrm{KCl}$ saturado a $37^{\circ} \mathrm{C}$. como electrólito do eléctrodo de referência e uma ponte de plasma I [12| entre o $\mathrm{KCl}$ e o sangue. O plasma é colocado num tubo cilíndrico e serve para evitar interferências devidas aos eriłróciıs.

\section{ELÉCTRODO DE VIDRO COMBINADO MEDIDOR DE $p H$ E A JUNÇÄO LÍQUIDA}

A célula l acima indicada, re quer o uso de dois cléctrodos para sc medir o pH. Na maior partc das situaçōes prálicas. แısa-se um cléctrodo de vidro sensivel ao $\mathrm{H}^{+}$e mede-se a dilercença de potencial entre este e 
um clécirodo de referência, quando mergulluados na soluçāo radrăo e. numa seyunda operaçāu. na soluçăo em estudo. Conrudo, mais frequentemente ainda. usa-se o Eléctrodo de vidro combinado medidor de $\mathrm{pH}-$ Iig. 2, en que o elécrodo da referência externo, de $\mathrm{Ag} / \mathrm{AgCl}$ é incluido na mesma haste que o cléctrodo de vidro propriamente ditu.

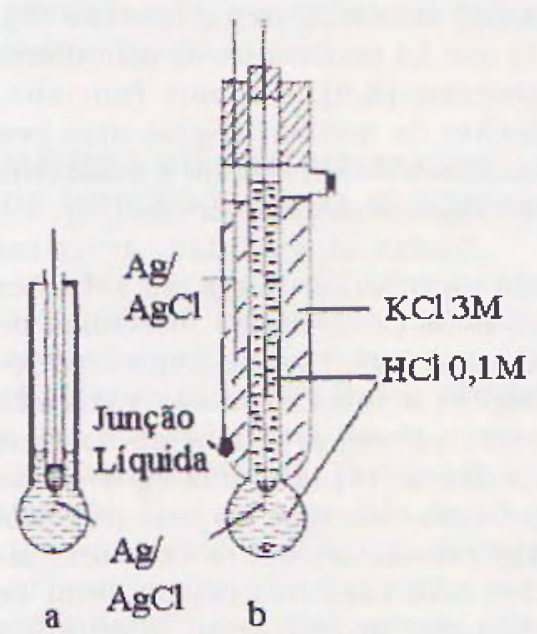

Fin 2 - Electrodus de sidro medidorss de $\mathrm{pH}$; - a - simples; b - combinado.

Deve-se notar que este eléctrodo de relerência cxtemo contém o seu electrólito igcralmente KCI $3 \mathrm{M}$ ou saturadoi num compartimento estanyue relativamente ąı compartimento onde se encontra a soluçăo interna do ejéctrodo dé vidro (que é. na matoria dos casos, $\mathrm{HCl} 0, \mathrm{l}, \mathrm{M}$ ). É de пotar ainda que no interior da membrana de vidro, mergulhado na soluçăo interna se encontra ainda outro eléctrodo de relerência interno de $\mathrm{Ag} / \mathrm{AgCl}$.

importa realçar que o eléctrodo de referência extcrno deve comunicar com a suluçăo em estudo estabele cendu a junçāo híquida necc'ssária ao luncionamento da célúla. Esta junçăo geralmente ocorre numa pequena placa de cerămica que está ajustada a un pequeno orificio na parede lateral do vidro colocado imediatamente acima da membrana de vidro especial que é sensivel ao $\mathrm{H}^{-}$. embora haja ou- tras tormas de se estabelecer essa jum. \{̧ăo, Este pormenor importante é lamentavelmente omitido em muitos livros!!

Dopartanemo de Quimia Bioquimia da FC'LL Facildade de Cinncias de fispog Camp Grande-1759-016 Lisbod

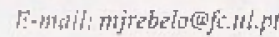

\section{BIBLIOGRAFIA}

1. R.C. Bates, E.A. Guggenleim, Purc Appl Chem. 1(1960)163.

2. Mark Jose F. Rebelo, Ply. D. Thesis "Sludy of factors influencing the precision of pllt measurcments" Newcastle-upon-Tyne, 198 !

3. A.K. Cuwington. Recent tles elopments in $\mathrm{pH}$ standardisation and medsuremenf for dilute aqutuos solulions. Anat. Chim. Ach. $127(198) 1,-21$

\$. A.K. Covington, R.C. B.ates and R. Durst. Pune and Appl. Chem. 57119851531

5.A.K. Covington, M.J.F, Retoto Determination of $\mathrm{pH}$ vilues over the lemperature range $5-60^{\mathrm{W}} \mathrm{C}$ for soms operational roforence standard soluti- ons and values oi the comentional residual Ifyuid juaction ponntials. And', Chim. Acts 2010!1987245-260.

6. D.R. Lide,ed. Hamdbook oi Chenistry and Physics, 7 git ed b-35, $(1997-1998$ ).

7. E.A. Gupgenheim, 1. Ar. Chen, Soc.. $52 \div 1930) 13: 5$

8. M.J.F. Reluelo. Liquid-liquid junction potuntials between brumide solutions. Electrochim: Acta 35(2)11990) 371

9. M.J.F. Retxalt Liguid junction potentials bolween $\mathrm{pH}$ luffer solutions, Purt. Electrorh. Acta, $817990) 61$.

10. C.R.R. Oliveira, M.J.F. Rebelu, M.F.G.F.C Camōes. Stability of liquid junction potentials. Piort. Flectroch. Acila. 91991253.

11. W. Davison, T.R. Harbinson, I'trformance of reference electrutcs with tree -difiusion junctiกกร, Mnal . Chim. Acla, 187(1986)55.

12. A.H.J. Maas, H.F. Weisslyerg, R.W. Burnelt O. Müllicr-I'lathe, P.D. Wimberley, W. G. ZijlsIra, R.A. Durst, O. 5iggdard-Amdersen, Reicrance Method for $\mathrm{pH}$ measurement in blend, f. Clin Chers. Chin. Biochur. 25 $1987 \mid 2 B 1-4$.

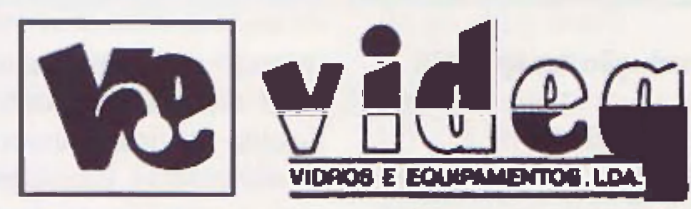

Equipamento de Laboratório

Balanças - Centrifugas - Aparelhos de $\mathrm{pH}$ - Tituladores Condutimetros - Agitadores - Espectrofotómetros Microscópios - etc.

Vidros e Plásticos de Laboratório Distribuidores NORMAX

\section{Material Didáctico}

Ensino Secundário e Superior

Representantes exclusivos SISTEDUC - Sistemas Educativos S.A

Fua Soeiro Pereira Gomes, 15 ric Frenle Bom Sucesso - 2615 Alverca

Telefs. (01) 95704 20/ $/ 2$ - Fax (351-1-957 04 23) - Portuga 